\title{
The T-box factor MLS-1 acts as a molecular switch during specification of nonstriated muscle in C. elegans
}

\author{
Stephen A. Kostas ${ }^{1,2}$ and Andrew Fire ${ }^{1,3}$ \\ ${ }^{1}$ Department of Embryology, Carnegie Institution of Washington, Baltimore, Maryland 21210, USA; ${ }^{2}$ Graduate Program \\ in Biology, The Johns Hopkins University, Baltimore, Maryland 21218, USA
}

We have isolated mutations in a gene $\mathbf{m l s - 1}$ that is required for proper specification of nonstriated muscle fates in Caenorhabditis elegans. Loss of MLS-1 activity causes uterine muscle precursors to forego their normal fates, instead differentiating as vulval muscles. We have cloned $\mathrm{mls}-1$ and shown that the product is a member of the T-box family of transcriptional regulators. MLS-1 acts as a cell fate determinant in that ectopic expression can transform other cell types to uterine muscle precursors. Uterine muscle patterning is executed by regulation of MLS-1 at several different levels. The mls-1 promoter is activated by the C. elegans orthologs of Twist and Daughterless, but is only active in a subset of the lineage where these two transcription factors are present. $\mathrm{mls}-1$ activity also appears to be regulated by posttranscriptional processes, as expression occurs in both uterine and vulval muscle precursors.

[Key Words: Muscle; myogenesis; T-box; asymmetric cell division; Twist; C. elegans]

Received June 25, 2001; revised version accepted November 16, 2001.

Contractile muscles comprise a functionally and morphologically diverse subset of mesodermal cells. Although the mechanisms underlying the early stages of mesodermal determination are increasingly well understood, the underlying basis of later myogenic diversity remains largely a mystery (for reviews, see Arnold and Braun 2000; Perry and Rudnick 2000). Two approaches to this problem have been pursued. First, experimental manipulations of myogenic precursor populations in Drosophila, vertebrates, ascidians, and Caenorhabditis elegans have shown the significance of lineally inherited cell identity as a fundamental component in fate specification (Nishida and Satoh 1983; Bate et al. 1991; Ordahl and Le Douarin 1992; Schnabel 1994). Second, a number of factors that have been implicated in general aspects of cellular signaling and specification have been tested for their effects on myogenic patterning (for review, see Baylies et al. 1998).

C. elegans provides a unique set of tools for the analysis of connections between lineage and cell fate because the effects of mutations on cell fate determination during development can be analyzed at single-cell resolution (Sulston and Horvitz 1977; Sulston et al. 1983). In particular, the postembryonic specification of mesoderm in C. elegans is an attractive system for studying the

${ }^{3}$ Corresponding author.

E-MAIL fire@ciwemb.edu; FAX (410) 243-6311.

Article and publication are at http://www.genesdev.org/cgi/doi/10.1101/ gad.923102. integration of myogenic determination and cell fate decisions. Mesoderm formed during hermaphrodite postembryonic development is derived from a single mesoblast $\mathrm{M}$, which gives rise to six cell types: striated bodywall muscles (14 cells), nonmuscle coelomocytes (2 cells), and four classes of nonstriated sex muscles (4 cells of each class). The M lineage provides a valuable microcosm for genetic and experimental manipulations of mesodermal patterning because the resulting cells are not essential for viability or for the overall body plan of the animals (Sulston and White 1980). Additionally, there are cell-specific transgene reporters for each cell fate within the postembryonic mesoderm that allow the facile identification of cell type (Corsi et al. 2000).

More than a dozen regulatory factors are known to be important for early proliferation and patterning of the $M$ lineage and for cell fate decisions between striated muscle, coelomocyte, and proliferating myoblasts (sex myoblasts) (Greenwald et al. 1983; Kenyon 1986; Harfe et al. 1998a,b; Corsi et al. 2000; Liu and Fire 2000; J. Liu, J. Yanowitz, S. Kostas, and A. Fire, unpubl.; C. Branda, and M. Stern, pers. comm.). These include putative transcription factors, signaling molecules, and a variety of factors with as yet unknown biochemical functions.

During the earliest stages of the M lineage, two members of the C. elegans Hox cluster (lin-39 and mab-5) have essential and overlapping roles in diversification of the postembryonic mesoderm (Liu and Fire 2000). Further analysis has shown that there are multiple targets of lin-39 and mab-5 activity in the $\mathrm{M}$ lineage, one of which is the C. elegans twist homolog, hlh-8 (Liu and Fire 
2000). hlh-8 is expressed in all undifferentiated cells within the lineage, and a null mutation in hlh-8 disrupts the specification of $\mathrm{M}$-derived striated and nonstriated muscles (Harfe et al. 1998b; Corsi et al. 2000). Additionally, hlh-8 is required for the expression of several genes active within the nonstriated musculature (Harfe et al. 1998b; Corsi et al. 2000; S. Kostas and A.Fire, unpubl.). CeTwist has been shown to cooperate with the C. elegans daughterless homolog CeE/Da (the product of the hlh-2 gene) in target activation (Harfe et al. 1998b). hlh-2 is expressed in a dynamic pattern throughout embryonic and larval development, including in the $\mathrm{M}$ lineage (Krause et al. 1997). Once the initial divisions of the M blast cell have occurred, a primary cell fate decision must distinguish striated muscle, coelomocyte, and sex myoblast fates. C. elegans homologs of the HLH transcription factor MYOD and the intercellular signaling factor Notch have been implicated in this decision. Lossof-function mutations in $h l h-1$, which encodes the $C$. elegans homolog of MyoD, result in conversion of coelomocytes to sex myoblasts, whereas loss-of-function mutations in the Notch homolog lin-12 show an inverse transformation (Greenwald et al. 1983; Krause et al. 1990; Harfe et al. 1998a).

Late in the $M$ lineage, there is a second set of cell fate choices between muscle cell types. Each of the two sex myoblasts produces four different nonstriated muscle types: two of these (vm1 and vm2) make up the vulval musculature, and two (um1 and um2) make up the uterine musculature. These cell types can be readily distinguished by morphology and by the expression of a set of green fluorescent protein (GFP) reporter transgenes, providing an excellent system to address the specification of distinct muscle fates.
In this report, we describe a genetic screen for mutations that alter the specification of uterine and vulval muscle fates, and the identification of a gene, mls-1 (muscle lineage specification-1), required for patterning in the $\bar{M}$ lineage. A loss of $m l s-1$ function leads to a specific cell fate transformation of uterine muscle precursors to vulval muscle precursors, whereas ectopic expression can produce a reverse fate transformation toward uterine muscle fate. mls-1 encodes a member of the T-box family of transcriptional regulators. The founding member of this family of DNA-binding proteins, Brachyury, or $\mathrm{T}$, is required for several aspects of mouse mesoderm specification (Herrmann et al. 1990; Beddington et al. 1992). There is a large family of transcriptional regulators that share a conserved DNA-binding domain (Tbox) with Brachyury (Pflugfelder et al. 1992; for review, see Smith 1999). mls-1 is most closely related to a subfamily of T-box genes that includes human $t b x 1$, a gene implicated in velocardiofacial/DiGeorge syndrome (Jerome and Papaioannou 2001; Lindsay et al. 2001; Merscher et al. 2001).

\section{Results}

A GFP-based genetic screen for altered specification of nonstriated muscle

During postembryonic development, the C. elegans hermaphrodite produces four distinct types of nonstriated muscle (Fig. 1). To isolate mutations that affect nonstriated muscle patterning, we carried out a fluorescencebased mutational screen using the GFP-expressing strain PD4251. GFP expression in PD4251 animals is driven by the myo-3 promoter and is present in all striated muscles
A

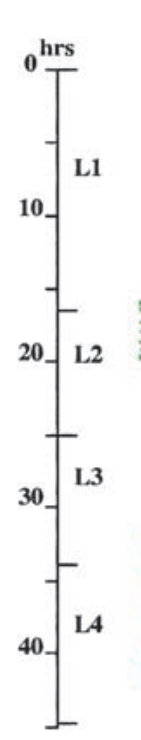

B

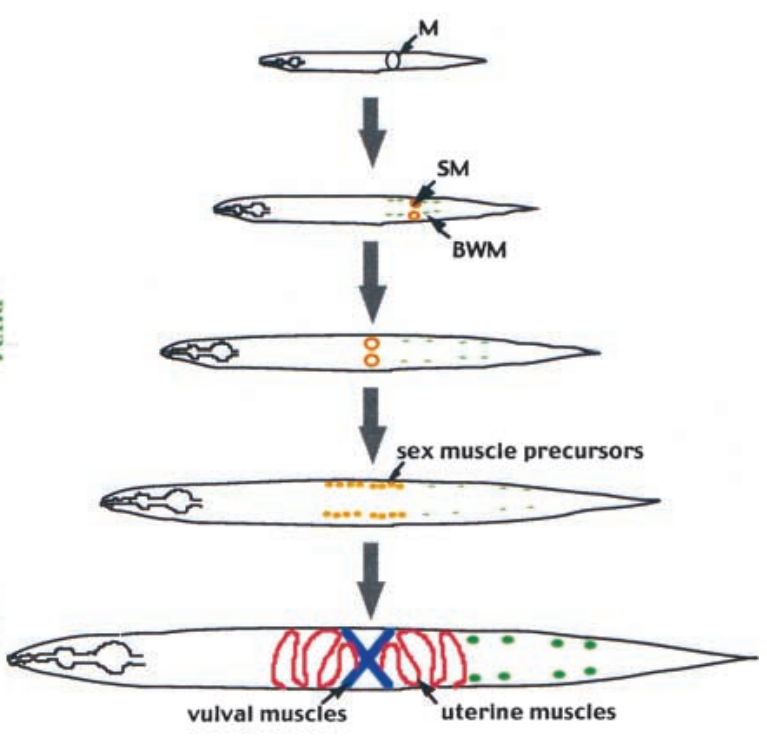

Figure 1. The C. elegans hermaphrodite postembryonic M lineage. Times indicated are $25^{\circ} \mathrm{C}$ posthatching (from Sulston and Horvitz 1977, redrawn from Liu and Fire 2000). (A) The M lineage with all differentiated cell types. (B) A schematic of the ventral view of the M lineage through larval development. 
and in a subset of nonstriated muscle cells. For our screen, the key property of PD4251 was the visual identification of the vulval musculature (Fig. 2A). Focusing on animals with supernumerary GFP-expressing cells in the vulval region, we obtained 14 independent mutant strains from a screen of 7000 mutagenized chromosome sets. Three mutations (cc569, cc570, and cc571) were chosen for further analysis: these show a transformation of cell fates within the nonstriated musculature (see below), whereas the remaining mutants from the screen appear to produce extra vulval muscles through an earlier shift in cell lineage (the production of supernumerary sex myoblasts; Greenwald et al. 1983; Kenyon 1986; Harfe et al. 1998a; S. Kostas and A. Fire, unpubl.).

Animals homozygous for cc569, cc570, or cc571 each had a similar pattern of myo-3::gfp expression: supernumerary muscles in the midventral region of the animal that appeared to be ectopic vulval muscles. The three mutations failed to complement as trans-heterozygotes, and all mapped to the central portion of Chromosome III (see Materials and Methods). The corresponding gene was designated $\mathrm{mls}-1$. mls-1 mutations produce a transformation of uterine to vulval muscles

The C. elegans sex muscles are the last muscles born during wild-type development (Fig. 1). They arise from the postembryonic $M$ mesoblast, which also produces 14 striated body-wall muscles and 2 coelomocytes (a nonmuscle cell). To determine the nature of the cell fate transformations we examined cell number and morphology using several molecular markers for terminally differentiated vulval muscle fate.

egl-15 encodes an FGF receptor. A stably integrated eg1-15::gfp transgene is expressed in the four Type 1 vulval muscles (vm1s): one in each of the four egg-laying muscle quadrants (Fig. 2C; DeVore et al. 1995; Harfe et al. 1998a). mls-1 mutant animals showed a doubling of eg1-15::gfp-expressing cells at the vulva (Fig. 2D). Of the three $\mathrm{mls}-1$ alleles, cc569 showed the highest expressivity (doubling of egl-15::gfp-positive cells in $97 \%$ of quadrants), whereas cc570 and cc571 showed slightly lower expressivity (doubling in $86 \%$ and $90 \%$ of compartments, respectively). Normal vm1 muscles are diagonal
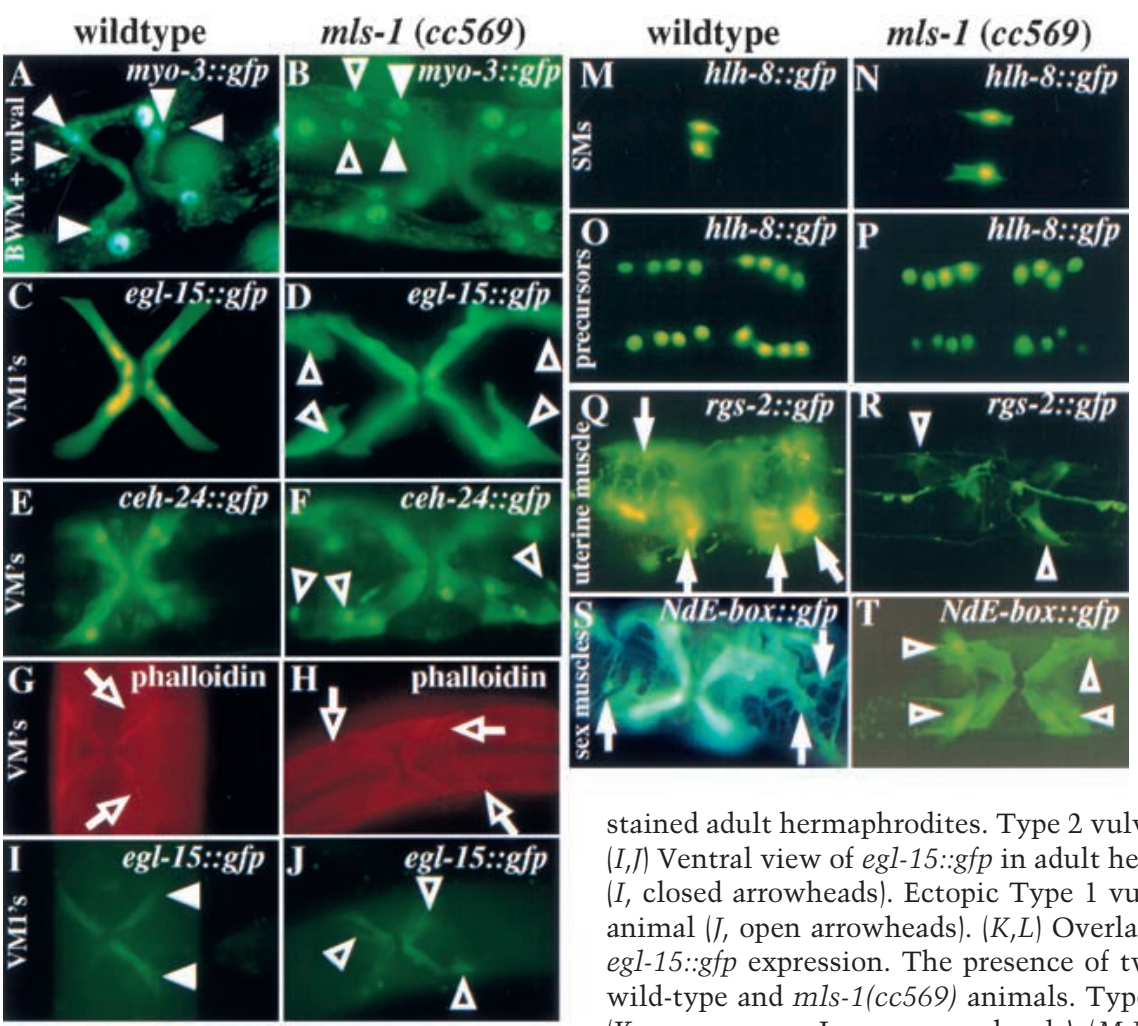

Figure 2. Mutations in $m l s-1$ produce a transformation of uterine muscles to vulval muscles. Fluorescence illumination photomicrographs of GFP reporters analyzed in wild-type $(A, C, E, G, I, K, M, O, Q, S)$ and $m l s$ 1(cc569) $(B, D, F, H, J, L, N, P, R, T)$ animals. The ectopic vulval muscle cells in mls-1 mutants are shown $(B, D, F, J, L, R, T$, open arrowheads). $(A, B)$ Ventral view of myo$3:: g f p$ in adult hermaphrodites. mls-1 alleles were isolated for their supernumerary myo-3::gfp-expressing cells. Nonectopic vulval muscles are indicated with closed arrowheads. $(C, D)$ Ventral view of egl$15:: g f p$ in adult hermaphrodites. $(E, F)$ Ventral view of ceh-24::gfp in adult hermaphrodites. Ectopic vulval muscles are shown in two quadrants $(F$, open arrowheads) Two sex muscle quadrants are out of the plane of focus. $(G-L)$ Ventral view of adult hermaphrodites in which the muscle actin structure and egl-15::gfp expression are visualized. $(G, H)$ Ventral view of phalloidinstained adult hermaphrodites. Type 2 vulval muscles are indicated $(G, H$, open arrows) $(I, J)$ Ventral view of egl-15::gfp in adult hermaphrodite showing Type 1 vulval muscles ( $I$, closed arrowheads). Ectopic Type 1 vulval muscles are shown in the mls-1(cc569) animal (J, open arrowheads). $(K, L)$ Overlaid photographs of muscle actin staining and eg1-15::gfp expression. The presence of two classes of vulval muscles can be seen in wild-type and mls-1(cc569) animals. Type 1 and Type 2 vulval muscles are indicated (K, open arrows; L, open arrowheads). ( $M, N)$ Ventral view of hlh-8::gfp expression in L2 hermaphrodite larvae. Wild-type and $\mathrm{mls}-1$ animals have two sex myoblasts, indicating that the ectopic vulval muscles in mls-1 mutants did not arise from production of additional sex myoblasts. $(O, P)$ Ventral view of twist::gfp expression in L4 larvae showed that the total number of sex muscle precursor cells was unchanged. $(Q, R)$ Uterine muscle fate is observed with $r g s-2:: g f p$ in ventral view of adult hermaphrodites. Within the sex muscles, $r g s-2:: g f p$ is more prominently expressed in uterine muscle $(Q$, closed arrows) than vulval muscle. Only faint vulval muscle expression of $r g s-2:: g f p$ was detected in $m l s-1(c c 569)$ animals $(R$, open arrowheads). $(S, T)$ Ventral view of $N d E$-box::gfp in adult hermaphrodites. The morphology of uterine muscle $(S$, closed arrows) and vulval muscle ( $T$, open arrowheads) can be distinguished. mls-1(cc569) animals lack rgs-2::gfp+ cells with uterine muscle morphology. 
and attach at one end to the lateral body wall and at the other to the vulval epidermis. Two different patterns were evident in mls-1 mutant animals: Type I, two parallel diagonal cells, each showing normal vm1 morphology (lateral body wall and vulval epidermis attachment), or Type II, one normal vm1 and a second cell that appeared similar but lacked the ventral vulval attachment. For young adults (before the onset of fertilization), mls1(cc569) showed 36\% Type I attachment pattern. The fraction of Type I cells appeared to decrease with age (15\% in older adults), suggesting that the lack of ventral attachment in the second cell in the Type II pattern may reflect a detachment as a function of age or use.

We used two markers that specifically label both vm1 and vm2 muscles; these were GFP fusions to ceh-24 (an NK class homeodomain; Harfe and Fire 1998) and arg-1 (a putative notch ligand; Fitzgerald and Greenwald 1995). Although each construct also expresses in several additional mesodermal cells of distinct morphology located far away from the vulva, the patterns overlap only in the vm1 and vm 2 cells. mls-1 mutant adults showed doubling of both ceh-24::gfp- and arg-1::gfp-positive cells at the vulva (Fig. 2E,F; data not shown). This suggests a doubling of both vm1 and vm 2 cells.

Two markers were used to probe the muscle character of the ectopic vulval-muscle-like cells. The original marker used in the screen (myo-3::gfp) is expressed in a broad set of nonpharyngeal muscle cells (both striated and nonstriated; Ardizzi and Epstein 1987); in addition, we examined actin filament structures in the cells using fluorescently labeled phalloidin for visualization (Fig. $2 \mathrm{~A}, \mathrm{~B}, \mathrm{G}-\mathrm{L})$. Both markers provide confirmation that the supernumerary cells are, indeed, muscles, with the phalloidin pattern (an extended single-sarcomere structure) consistent with a vulval muscle character. Double marking with phalloidin and egl-15::gfp also provides a means to confirm the presence of putative vm2 cells in the mls-1 mutant (Fig. 2K,L).

We considered several possible origins of the extra vulval muscles (VMs). We examined the entire $M$ lineage throughout larval development using an hlh-8::gfp transgene (Fig. 2M-P). hlh-8 encodes the C. elegans Twist homolog and is expressed in all undifferentiated cells from the M lineage (Harfe et al. 1998b). hlh-8::gfp provides a valuable marker for examining $M$ lineage patterns in individual larvae and in populations of animals (Harfe et al. 1998b). We saw no defects during the initial phases $(\sim 30 \mathrm{~h})$ of the $\mathrm{M}$ lineage in mls-1 mutants (Fig. $2 \mathrm{~N}, \mathrm{P})$. Both wild-type and mls-1 mutant L2 animals produced two sex myoblasts, indicating the extra vulval muscles in mls-1 mutants did not arise from the production of additional sex myoblasts (Fig. 2M,N). Wild-type L4 animals expressing hlh-8::gfp have 16 sex muscle precursors arranged in quadrants of 4 around the vulva (Fig. 2O). mls-1 mutant animals had the same number and pattern of hlh-8::gfp-expressing cells, indicating the total number of M-derived body-wall muscles, coelomocytes, and sex muscle precursors was conserved between mls-1 mutant and wild-type animals (Fig. 2P).

Because the number of $\mathrm{M}$-derived cells and their pat- tern of divisions were conserved in mls-1 mutant animals, we tested whether the extra VMs resulted from a transformation of uterine muscle to vulval muscle. We examined mls-1 mutants for cells with uterine muscle fate using an integrated rgs-2::gfp transgene (Dong et al. 2000). rgs-2 encodes a G-protein regulator expressed in several tissues, with very strong expression in the uterine muscles and much weaker expression in vulval muscles (Fig. 2Q). mls-1 mutant animals carrying an rgs2::gfp transgene produced a strong GFP signal in all tissues that normally express the transgene, with the notable exception of uterine muscle (Fig. 2R). Thus, although hlh-8::gfp expression showed the uterine muscle precursors are made, $r g s-2:: g f p$ expression indicated that the cells fail to adopt a uterine muscle fate. This suggested the production of extra vulval muscle in mls-1 mutant animals occurred at the expense of related uterine muscle cells.

Additional confirmation of the transformation of uterine muscle to vulval muscle has come from the analysis of a marker NdE-box::gfp that is expressed in all mature sex muscles (Harfe et al. 1998b). mls-1 mutant animals had the same number of NdE-box::gfp-expressing cells as wild type (Fig. 2S,T). Cells fated to become uterine muscle in wild-type animals showed several characteristics of vulval muscle in mls-1 mutant animals, including morphology, position, and attachments that closely resembled vulval muscle. Using mls-1 mutant animals carrying both hlh-8::gfp and NdE-box::gfp, we were able to follow cells as they differentiated from precursor cells to sex muscles. In wild-type animals, the uterine muscles ingress, form thin sheets that spread out on the uterus, and attach to the body wall and uterus (Sulston and Horvitz 1977). In mls-1 mutants, the cells did not ingress, but formed tubular muscles indistinguishable from vulval muscles, and were able to attach to the vulva and body wall.

Lineages producing a variety of other striated and nonstriated muscle fates were apparently normal in $\mathrm{mls}-1$ mutant animals. Using the myo-3::gfp transgene (see above), we characterized the body-wall muscle pattern and found no detectable differences from wild type. Enteric muscle morphology appeared identical to wild type when observed using arg-1::gfp. Enteric muscle function is required for defecation, and disruption of enteric muscle function can result in constipation (Thomas 1990). mls-1 mutants were not constipated and appeared to have normal defecation behavior, suggesting that mls-1 is not essential for patterning the enteric muscles. The head mesodermal cell was observed with $\arg -1:: g f p$. Both position and morphology of the head mesodermal cell appeared normal in mls-1 mutants (data not shown).

mls-1 encodes a C. elegans homolog of vertebrate TBX1 factors

The mls-1 locus was mapped to an interval spanned by mec-14 and lin-39 on Chromosome III. DNA clones from this region were injected into mls-1 mutant animals, and progeny were assayed for rescue of the sex 
muscle phenotype. One YAC (Y69F12) and one fosmid (H14A12) rescued mls-1. H14A12 contains seven predicted open reading frames. Injection of H14A12 subclones revealed a 4.4-kb fragment sufficient for mls-1 rescue (Fig. 3A; see Materials and Methods). This fragment contains a single open reading frame, H14A12.4. Insertion of 4 bases within the H14A12.4 reading frame (predicted to truncate the protein after 104 amino acids) eliminated the rescuing activity of the clone (Fig. 3A).

H14A12.4 is predicted to encode a 252 -amino-acid protein containing a 187-amino-acid T-box domain (Consortium 1998). H14A12.4 is one of at least 19 TBX family members in C. elegans (Ruvkin and Hobert 1998). Alignment of H14A12.4 with its closest relatives as well as with mouse Brachyury is shown in Figure 3C. H14A12.4 is most similar to a subfamily of T-box factors that includes human TBX1 (Fig. 3D). Within the T-box domain, the predicted mls-1 product is $55 \%$ identical and $71 \%$ similar to human TBX1. Homology between H14A12.4 and other T-box factors is limited to the T-box domain.

\section{Loss-of-function character of mls-1 alleles}

To determine the molecular nature of the mls-1 alleles, the H14A12.4 genomic sequence was obtained from each of the mutants. The three mutations each had a distinct single base-pair defect. mls-1(cc569) had a G $\rightarrow$ A transition in the predicted splice acceptor of the last exon, mls-1(cc571) had a $\mathrm{T} \rightarrow \mathrm{A}$ transversion in the coding region within the fourth exon, and $m l s-1(c c 570)$ had a
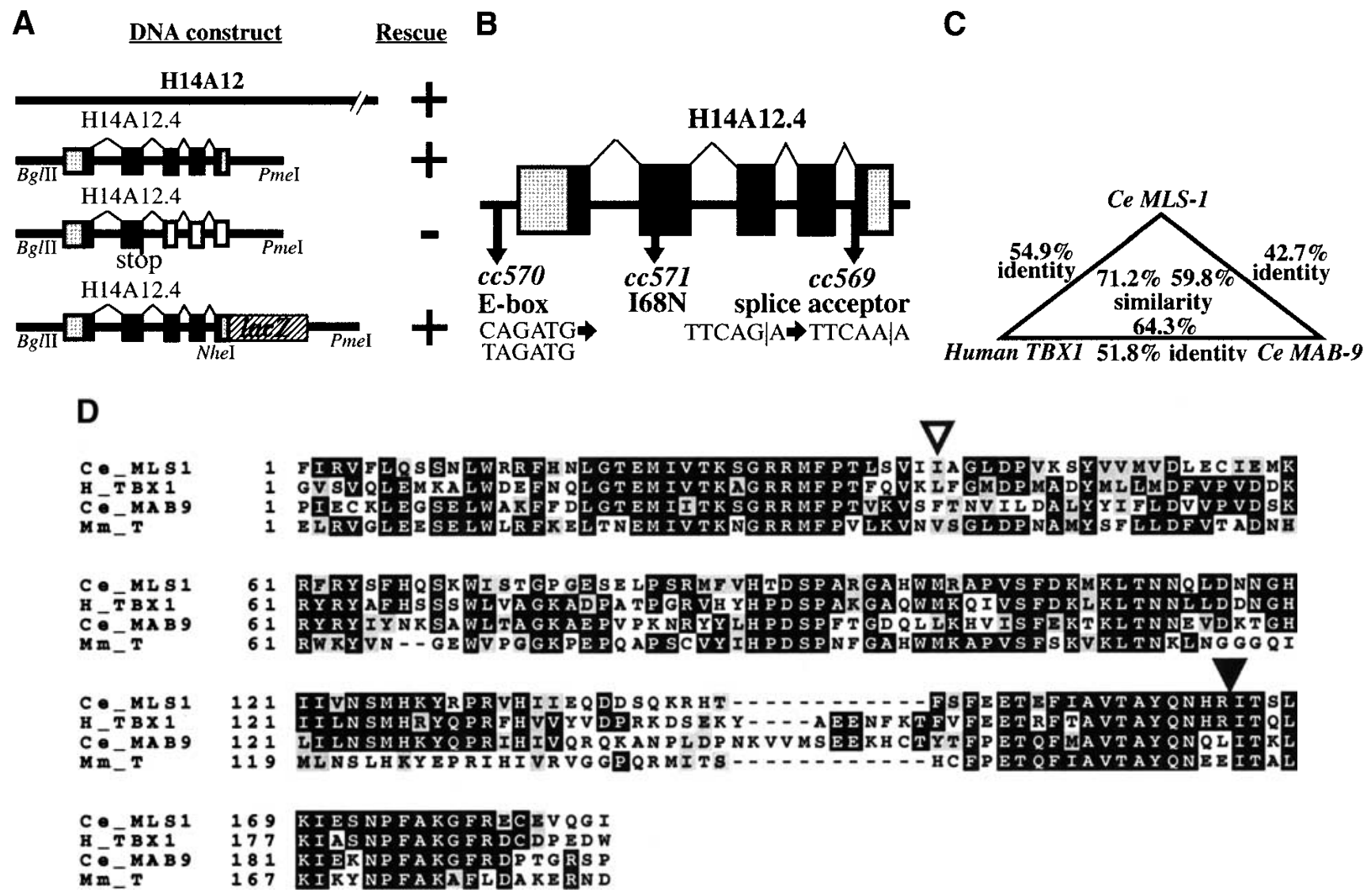

Figure 3. Cloning mls-1. (A) (upper segment) A 4.4-kb BglII-PmeI subclone from fosmid H14A12 was able to rescue all mls-1 alleles. This fragment contains a single coding sequence as predicted by GeneFinder (P. Green, unpubl.). The T-box DNA-binding domain is colored black. (Middle segment) A clone containing an insertion at an AgeI site in the second exon causes a truncation at amino acid 109. The resulting sequence fails to rescue. (Lower segment) The structure of the $m 1 s-1:: 1 a c Z$ transgene is shown. LacZ was fused six amino acids from the C terminus of MLS-1. The construct results in the loss of the C-terminal six amino acids of MLS-1, but has the mls-1 3' untranslated sequence. The mls-1::1acZ construct retains rescuing activity. (B) The mutations in mls-1 alleles cc569, cc570, and cc571 are shown. (C) Sequence comparison of CeMLS-1, human TBX1 (Chieffo et al. 1997), and CeMAB-9 (Woolard and Hodgkin 2000|. Only residues within the T-box domain were compared. The genes have no similarity outside the T-box domain. Amino acid similarity was determined using the BLOSUM62 matrix (Henikoff and Henikoff 1992). (D) Sequence alignment of T-box domains. The alignment of the amino acid sequence of the T-box domain is shown for MLS-1 (Ce_MLS1), human TBX1 (H_TBX1; GenBank accession no. AF012130), C. elegans MAB-9 (Ce_MAB9; accession no. AJ252168), and mouse Brachyury (Mm_T; accession no. X51683). The start of the T-box domain of each protein is labeled 1. Identical amino acids are shown in black, similar amino acids are shaded. The MLS-1 sequence is based on GeneFinder prediction, homology-based sequence analysis, and a single allele defining the fourth splice acceptor. Positions of the mls-1 mutations in $c c 569$ (closed arrowhead) and cc571 (open arrowhead) are shown. 
$\mathrm{C} \rightarrow \mathrm{T}$ transition 179 bases upstream of the predicted coding region (Fig. 3B).

TBX factors are nuclear DNA-binding proteins that modulate gene expression through binding to specific control elements in target genes (for review, see Smith 1999). The crystal structure of the Xenopus Brachyury homolog is available and provides a starting point in interpretation of the effects of mutations (Muller and Herrmann 1997). mls-1(cc569) encodes a truncated protein that is missing 60 amino acids, 18 of which are part of the T-box DNA-binding domain. The truncated protein would be predicted to lose two $\alpha$-helices that form the major interaction face with the target DNA sequence (Muller and Herrmann 1997). The base change in mls1(cc571) would convert isoleucine 68 to asparagine. This site is a conserved hydrophobic residue in the TBX family. In XBRA, the corresponding amino acid resides in a $\beta$-sheet, with the hydrophobic side chain inserted into the core of the protein (Muller and Herrmann 1997).

cc569 is the most severe of the mls-1 alleles, with several lines of evidence suggesting that the sex muscle transformation phenotype is a strong loss-of-function or null phenotype. First, this allele would be expected to produce a protein with little or no DNA-binding activity. Second, the cc569 allele is fully recessive (as are the other two alleles). Third, cc569 behaved as expected for a strong loss-of-function or null mutation in that the specification phenotype was not changed when placed in trans to several deficiencies for the region $(n D f 16$, $n D f 17)$. RNA interference (RNAi) experiments further suggest that the sex muscle cell fate transformation results from a reduction in $\mathrm{mls}-1$ function. Injection of specific double-stranded RNA (dsRNA) into adult animals is a particularly effective means for reducing gene function during embryogenesis in the next generation (Fire et al. 1998); no embryonic phenotypes were observed following parental injection of dsRNA corresponding to mls-1. Soaking of L1 larvae in dsRNA can also be used to reduce expression of specific genes during larval development (Tabara et al. 1998). Soaking of L1 larvae in dsRNA corresponding to a 546-bp fragment of mls-1 coding sequence phenocopied mls-1 mutant animals, but produced no additional overt phenotypes.

mls- 1 expression in sex muscle precursors coincides with segregation of uterine muscle fate from an asymmetric cell division

GFP and $\beta$-galactosidase reporter constructs were used to analyze the pattern of $\mathrm{mls}-1$ promoter activity during development. Transgenes in which the reporter was fused in frame at the C-terminal NheI site of the rescuing construct were found to retain their rescuing activity when present in extrachromosomal arrays: The mls$1:: g f p$ and $m l s-1:: 1 a c Z$ transgenes restored presumptive uterine cells to their proper fate, whereas presumptive vulval muscles retained a vulval muscle fate (Fig. 3A). The ability of the transgene to restore the wild-type uterine muscle pattern without disruption of other fates is consistent with some fidelity of these transgenes in reproducing the endogenous $\mathrm{mls}-1$ expression pattern.

The mls-1 transgenes were expressed in the sex myoblast lineage and in a subset of the enteric muscles. Expression of the mls-1 transgenes in the M lineage was detected as early as the sex myoblast granddaughters (M.vl/rpaaa/pa/p; Fig. 4A). These cells are the daughters of the asymmetric division that gives rise to uterine and vulval muscles. The transgene product was initially observed to accumulate in the uterine muscle progenitors and persisted throughout uterine muscle differentiation (Fig. 4B-E). In the initial period after segregation of uterine muscle and vulval muscle fate, the vulval muscle progenitor lineage lacked $\mathrm{mls}-1$ transgene activity (Fig. $4 \mathrm{~A}, \mathrm{~B})$. However, after division of the vulval muscle progenitors to Type 1 and Type 2 vulval muscle precursors, mls-1 transgenes were expressed in Type 2 vulval muscles (Fig. 4C-E). Expression of $\mathrm{mls}-1$ transgenes was not observed in Type 1 vulval muscles (Fig. 4D,E). mls-1 transgenes were also expressed in a subset of the enteric muscles (Fig. 4G). mls-1 was expressed in the left intestinal muscle (AB.plpppppaa), right intestinal muscle (MS.ppaapp), and the anal depressor muscle (AB.plpppppap). Expression was not detected in the sphincter muscle (AB.prppppap). The enteric muscles are required for defecation behavior, and certain mutations that disrupt their proper differentiation can result in constipation (Thomas 1990; Corsi et al. 2000). Defecation behavior appeared wild type in the mls-1 mutant animals and in $m l s-1$ (RNAi) animals, suggesting that $m l s-1$ is not necessary for intestinal and anal depressor muscle fate.

mls- 1 is a uterine muscle fate determinant

To test the sufficiency of $m l s-1$ in uterine muscle fate specification, we ectopically expressed $\mathrm{mls}-1$ in cells that normally do not become uterine muscle. As noted above, the $h l h-8$ promoter is active at high levels and for an extended time period in all undifferentiated cells from the $\mathrm{M}$ lineage, including body-wall muscle precursors, coelomocytes, and vulval and uterine muscle precursors. This promoter allowed us to test the effect of broad MLS-1 expression on several cell types within the postembryonic mesoderm.

We first assayed for the effect of misexpressed mls-1 on vulval muscle fate using an egl-15::gfp reporter. Vulval muscles were lost in animals expressing hlh-8::mls-1 (Fig. 5A-D). Striated body-wall muscle was visualized using the integrated myo-3::gfp reporter. In animals that carried the hlh-8::mls-1 construct, $64 \%$ of viable animals had a reduced number of $\mathrm{M}$-derived body-wall muscles (Fig. 5G,H). The non-muscle coelomocytes were visualized using a secreted GFP that is selectively taken up by coelomocytes (Harfe et al. 1998a). Wild-type animals have six coelomocytes, of which two are M-derived. Animals that misexpressed mls-1 were found to lack the two posterior, M-derived coelomocytes (Fig. 5I,J).

To definitively assess the cell fate effects of ectopic MLS-1 on the uterine musculature, we used an $\mathrm{mls}-1$ 


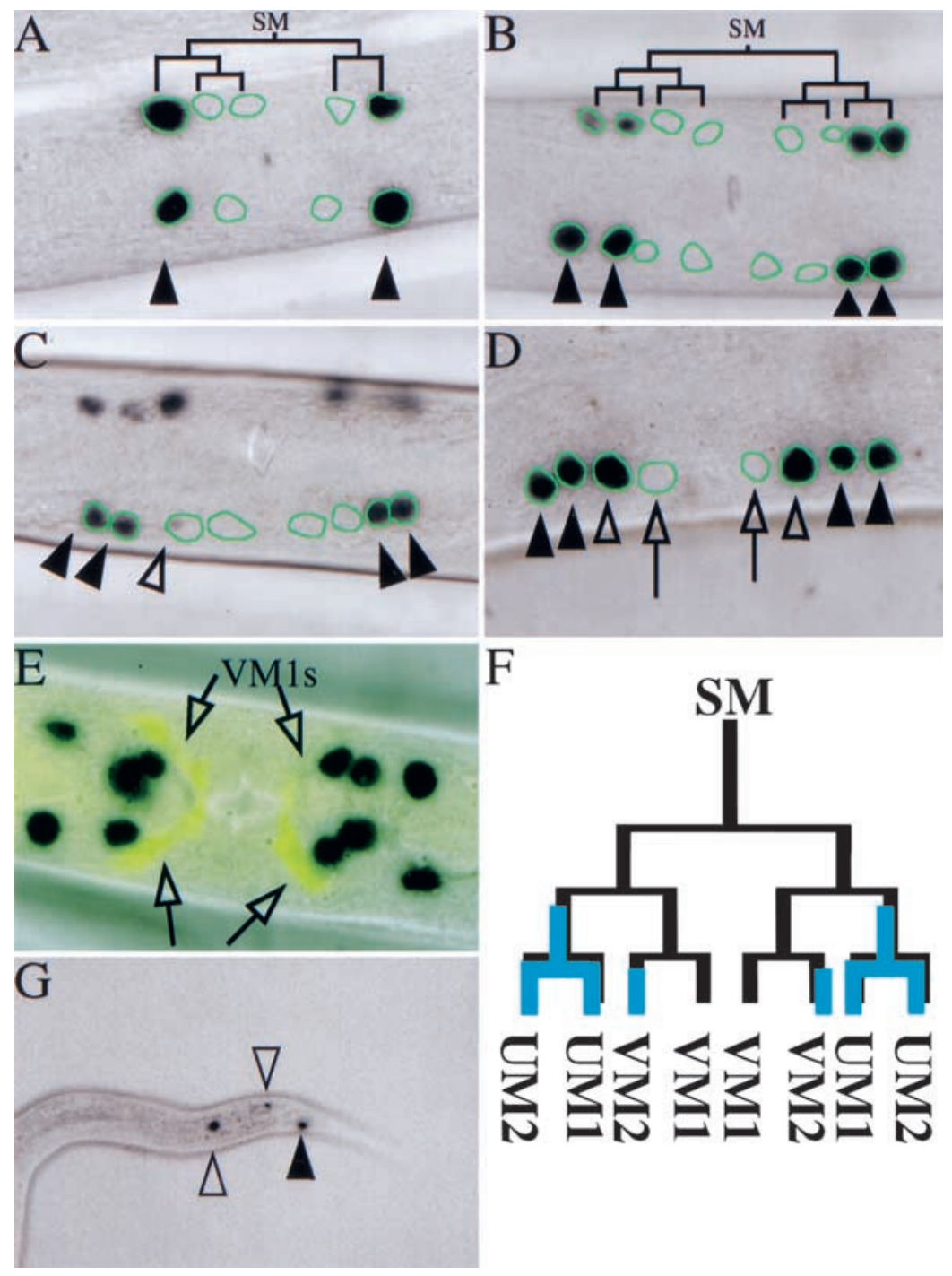

Figure 4. Expression pattern of mls-1::1acZ. Photomicrographs of expression pattern of a rescuing mls-1::1acZ transgene are shown in wildtype animals. The nuclei of sex myoblast-derived cells were traced using hlh-8::gfp expression (data not shown). $(A, B)$ The cell lineage is superimposed for descendants of one sex myoblast. $(A)$ Ventral view of L3 larval hermaphrodite. Expression in sex myoblast granddaughters is shown (arrowheads). (B) Ventral view of L4 hermaphrodite, soon after terminal division of sex myoblasts. $\mathrm{mls}-1:: 1 a c Z$ expression is observed in uterine muscle precursors only (arrowheads). $(C)$ Ventral view of L4 hermaphrodite. Onset of mls-1::1acZ expression in Type 2 vulval muscle precursors is shown (open arrowhead). (D) Lateral view of late L4 hermaphrodite showing $\mathrm{mls}-1:: 1 a c Z$ expression in uterine muscle (arrowheads) and Type 2 vulval muscle precursors (open arrowhead), but not Type 1 vulval muscle precursors (open arrows). (E) Ventral view of adult hermaphrodite. mls-1::1acZ expression was not observed in Type 1 vulval muscles (shown with egl-15::gfp, open arrows). (F) The wild-type sex myoblast lineage with overlay of mls-1::1acZ expression in blue. Wild-type animals have two sex myoblasts, with identical lineages. $(G)$ Lateral view of larval hermaphrodite tail. mls-1::1acZ expression in the anal depressor muscle (arrowhead) and intestinal muscles (open arrowheads). mutant background, which would otherwise lack uterine muscles. Expression of the hlh-8::mls-1 transgene restored uterine muscle fate in mls-1(cc570) animals, as evidenced both by the appearance of $r g s-2:: g f p$-positive cells in the uterine region, by the uterine-muscle-like morphology of these cells, and by positioning within the animal (Fig. 5E,F). The uterine musculature in mls1(cc570) animals expressing hlh-8::mls-1 was greatly expanded in comparison with wild type. The concomitant loss of several nonuterine muscle cell types from the $M$ lineage and appearance of supernumerary uterine muscle fates is strongly suggestive of a transformation in which some (if not all) of the absent mesodermal cell types from the $\mathrm{M}$ lineage had been converted by ectopic $\mathrm{mls}-1$ expression into uterine-type muscles.

Expression of mls-1 requires activation by the C. elegans Twist ortholog

Given that the expression pattern of $m l_{s-1}$ is a subset of the hlh-8 pattern and given that hlh-8 is required for the expression of numerous other markers in this lineage, we tested whether hlh-8 might serve directly to activate mls-1. Using a null mutant for hlh-8, we found that CeTwist was required for expression of a rescuing mls-1::1acZ transgene (Fig. 6A-C). Conversely, ectopic CeTwist could induce mls-1 expression. Because CeTwist had been shown to cooperate with the C. elegans Daughterless homolog $\mathrm{CeE} / \mathrm{Da}$ in activating target gene expression, we used a protocol in which a heat shock promoter was used to ectopically express a combination of CeTwist and $\mathrm{CeE} / \mathrm{Da}$ in animals carrying an mls-1::1acZ reporter transgene (Harfe et al. 1998b). Induction of CeTwist + CeE/Da produced a drastic increase in mls-1::1acZ expression compared with sibling controls (Fig. 6D). mls-1::1acZ expression in heatshocked animals was broadly and robustly expressed in tissues that do not normally express the transgene.

Several lines of evidence suggested that a site at -179 (relative to the ATG) in the mls-1 promoter may be a direct target for induction by hlh-8. The point mutation cc570 affects the -179 E-box and leads to a moderately strong reduction-of-function phenotype for $\mathrm{mls-1}$. Induc- 
Figure 5. MLS-1 is a uterine muscle fate switch. Fluorescence illumination photomicrographs of GFP reporters active in M-derived postembryonic mesoderm and analyzed in animals without ectopic mls-1 expression $(A, C, E, G, I)$ and with mls-1 expressed from the hlh-8 promoter $(B, D, F, H, J)$. Position of the vulva is indicated ( $B, D-I$, asterisk). The hlh- 8 promoter is active throughout the M lineage, allowing assay of MLS-1 function in cells normally programmed for nonuterine muscle fates. $(A-D)$ Expression of integrated egl-15::gfp in Type 1 vulval muscles of wild-type $(A)$ and $m l_{s-1(c c 570)}(C)$ animals. Expression is abrogated in the presence of ectopic mls-1 (B,D). (E) mls-1(cc570) animal expressing the integrated $r g s-2:: g f p$. Uterine muscle expression cannot be detected. (F) mls-1(cc570) animal with ectopic $m l s-1$ expression recovers $r g s-2:: g f p$ expression in uterine-muscle-like cells that often occupy positions posterior of the vulva. $(G, H)$ Lateral view of posterior half of wild-type animals with integrated myo-3::gfp expression in body-wall muscles. Body-wall muscle quadrants have eight muscles posterior of the vulva in wildtype $(G$, open arrowheads) and $m l s-1(c c 570)$ animals (data not shown). Animals with ectopic $m l s-1$ expression are often missing posterior body-wall muscles $(H)$. Fewer body-wall muscles can be visualized by the increased spacing between nuclei or by counting the cells. The animal shown has a quadrant with six body-wall muscles posterior of the vulva ( $H$, open arrowheads). $(I, J)$ Low magnification lateral view of animals expressing a secreted GFP that labels coelomocytes. Wild-type animals $(I)$ have six coelomocytes, including two in the dorsal posterior that are $\mathrm{M}$-derived ( $I$, arrowheads). Ectopic expression of mls-1 eliminates postembryonic coelomoctyes $(J)$.
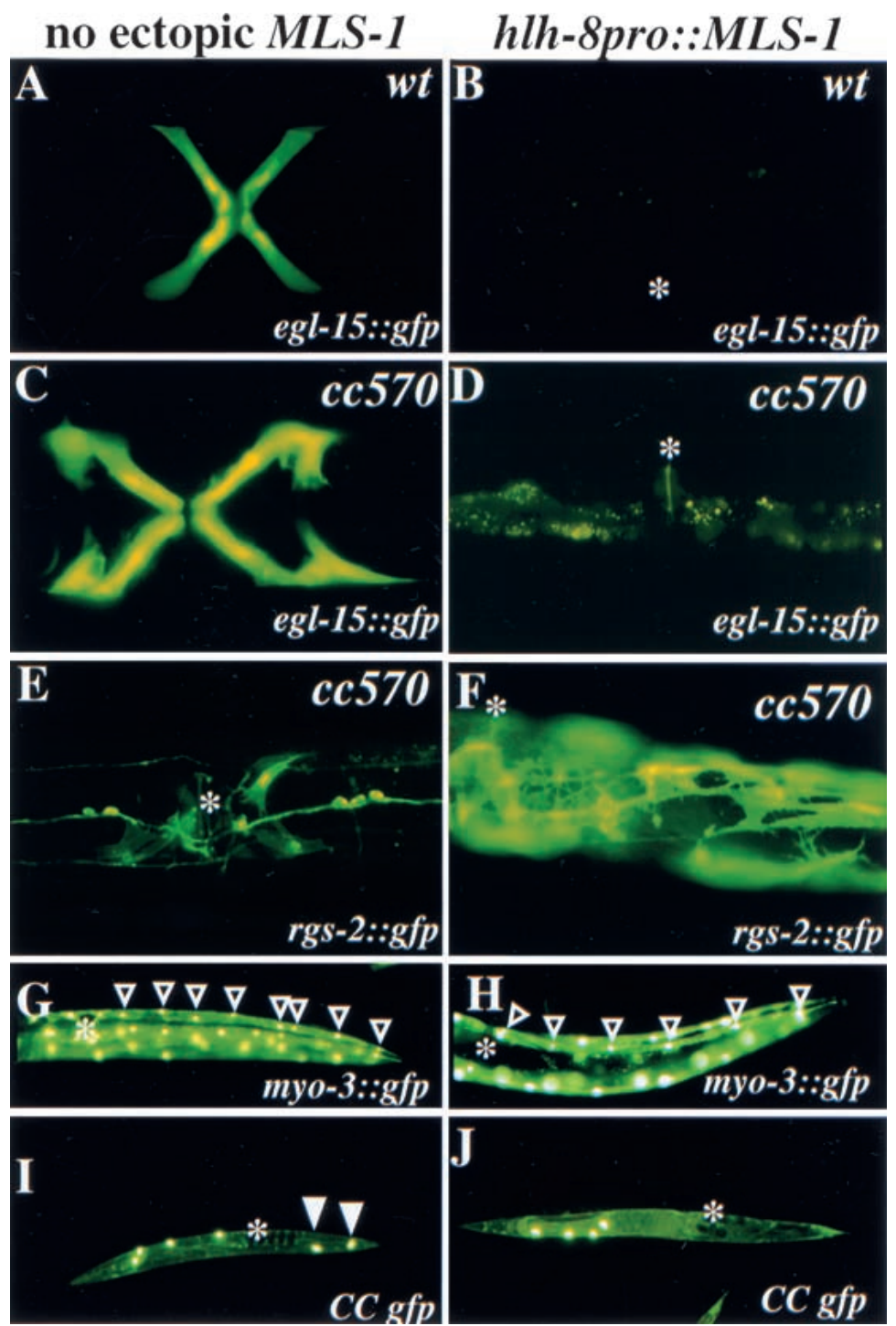

tion of mls-1::1acZ by CeTwist $+\mathrm{CeE} / \mathrm{Da}$ was also dependent on the -179 E-box in the mls-1 promoter (Fig. $6 E)$. The -179 site need not be the only interaction site for CeTwist in the mls-1 promoter: the -179 mutation cc570 has residual genetic activity, and reporter assays show a limited degree of hlh-8-dependent expression from constructs with a mutated -179 E-box (Fig. 6B,C,E). Two additional E-box sequences are located at positions
Figure 6. Activation of MLS-1 by CeTwist. $(A-C)$ Photomicrographs of $\mathrm{mls}-1:: 1 a c Z$ expression in wildtype $(A)$ and hlh-8(nr2061) $(B, C)$ adult hermaphrodites. $(B, C)$ No expression of mls-1::lacZ is seen in hlh-8 null animals. The animal shown in $C$ is older and therefore larger than those shown in $A$ and $B .(D, E)$ Photomicrographs of adult and L4 hermaphrodites after heat shock carrying an extrachromosomal array with pBH48.8 (heat shock promoter::CeTwist) and pKM1035 (heat shock promoter::CeE/Da). (D) Animals carry an extrachromosomal array with the rescuing mls-1::1acZ construct (pSAK534). (E) Animals carry an extrachromosomal array with an mls-1::1acZ in which the E-box at position -179 has been mutated to TAGATG $(\Delta-179$ E-box-ClaI).
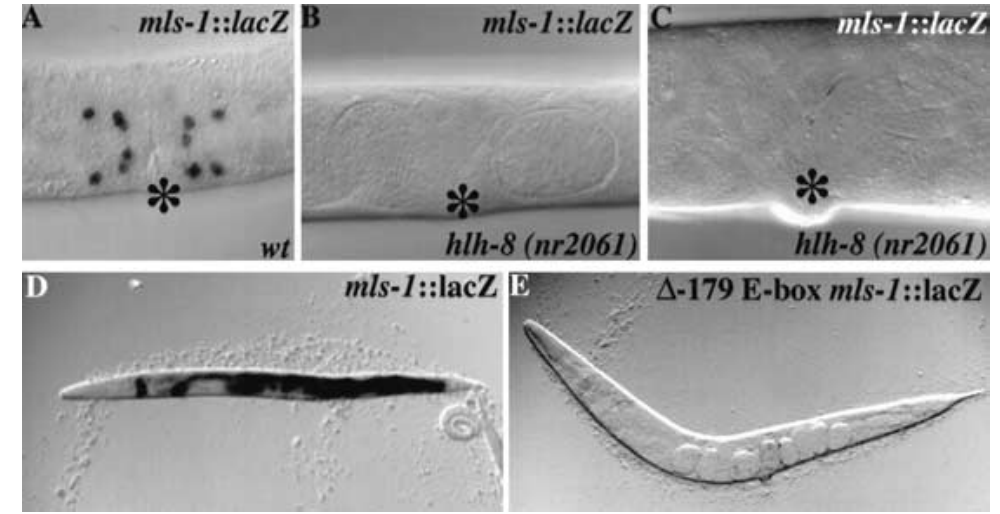
-123 and -213 ; these may suffice for partial activation in the absence of the -179 E-box.

A direct induction of $\mathrm{mls}-1$ by physiologic concentrations of CeTwist was suggested by a series of experiments in which alterations at the -179 site were assayed for compatibility with transgene-mediated rescue of $\mathrm{mls}$ 1(cc570). Mutations eliminating the -179 E-box resulted in a loss of rescue activity in transgenic lines, whereas a mutation that converts the E-box sequence to a perfect twist consensus sequence (CAtaTG) was compatible with rescue activity (Fig. 7). A further analysis of these transgene rescue experiments provided a surprising result: In the first generation following injection of DNA, all of the -179 E-box mutant constructs rescued $\mathrm{mls}$ 1(cc570), but (as noted above) rescue in subsequent generations required an intact E-box at this position. Previous analyses of transgene expression have suggested that $\mathrm{F}_{1}$ animals resulting from DNA injection carry singleunit DNAs with predominantly euchromatic character, whereas maintenance in subsequent generations involves extrachromosomal arrays with some heterochromatic properties (Okkema et al. 1993; Kelly et al. 1997; Hsieh et al. 1999). This suggests that hlh-8 binding the

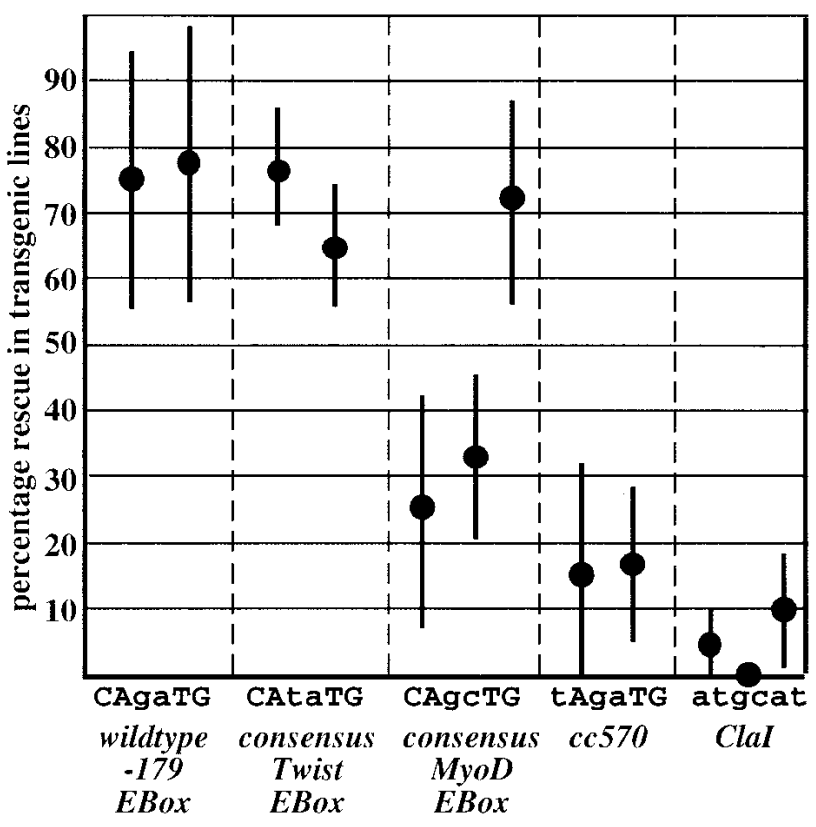

Figure 7. The -179 E-box is required for $m l s-1$ activity. A genomic segment carrying the mls-1 locus with different mutations in the -179 E-box was assayed in transgenic lines for rescue of uterine/vulval muscle fate decisions in an mls-1(cc570) mutant background. Each data point represents the mean percent rescue for an independent transgene array. Error bars give standard error of the mean. Rescue activity was strong in all transgenic lines with wild-type (CAgaTG) and Twist consensus (CAtaTG) E-boxes; little or no rescue was observed with nonE-box sequences cc570 (tAgaTG) or ClaI (atgcat). A consensus MyoD E-box (CAgcTG) yielded rescue in one of three transgenic lines; activation in this line might potentially involve Twist, MyoD, or another HLH factor, and has not been further analyzed.
-179 E-box sequence may function to overcome a chromatin-mediated repression of $\mathrm{mls}-1$.

\section{Discussion}

mls- 1 is required for specification of $\mathrm{C}$. elegans uterine muscle fate

We have described a GFP-based mutagenesis screen designed to isolate myogenic determinants and regulators of muscle patterning. Three mutations isolated in the screen were allelic and defined a new genetic locus, which we have named muscle lineage specification-1 $(\mathrm{mls}-1)$. mls-1 mutations result in a specific muscle defect in the postembryonic musculature: a loss of uterine muscle fate. The C. elegans postembryonic mesoderm is derived from a single blast cell, $M$. We have shown the $M$ lineage divisions are not disrupted in mls-1 mutant animals, nor is specification of other cell fates within the $M$ lineage. The mls-1 mutations caused no defects in embryonic striated muscle, postembryonic striated bodywall muscle, or the nonstriated gut-associated muscles. We show that the loss of uterine muscle fate is caused by a transformation of the precursor cells to an alternate nonstriated muscle fate, vulval muscle. This indicates that $\mathrm{mls}-1$ is not required for muscle determination, but is required for specific cell identity.

The role of $\mathrm{mls}-1$ in the postembryonic mesoderm may be limited to a single cell division in each quadrant of the $M$ lineage, because $m l s-1$ mutations do not disrupt a later fate decision, the specification of Type 1 and Type 2 sex muscle. The sex muscle transformation is evidently a loss of function (and perhaps null) phenotype, because mls-1(RNAi) and $m l s-1(c c 569) / D f 17$ animals show similar phenotypes with no additional defects in specification.

\section{Regulation of mls-1 activity}

The onset of mls-1 expression apparently coincides with the developmental decision in which the factor participates: the lineal segregation of uterine and vulval muscle progenitors. The CeTwist homolog, $h l h-8$, is a critical activator of $\mathrm{mls}-1$ expression. hlh- 8 activity precedes and is necessary for mls-1 expression. Under certain conditions, activity of $h l h-8$ (and of a cofactor CeE/Da) is sufficient for $\mathrm{mls}-1$ expression: forced expression of the hlh-8 product with CeE/Da was sufficient to drive ectopic expression of $\mathrm{mls}-1$ in numerous tissues. Sequence requirements suggested a direct interaction: the ability of $h l h-8$ to efficiently activate $m l s-1$ expression was dependent on a canonical HLH binding site 179 nucleotides upstream of the coding region.

Several observations suggest the activity of $m l_{s-1}$ in the $M$ lineage must be tightly regulated. We have shown that ectopic expression of mls-1 is not compatible with the other M-derived cell fates. Animals that misexpress mls-1 showed a loss of vulval muscles, body-wall muscles, and coelomocytes. A simultaneous increase in 
uterine muscles suggested ectopic mls-1 expression can transform M-derived cells to uterine muscle precursors. Such an activity needs to be definitively limited to specific cells that require its function (in this case, uterine muscles). At some level this may be enforced by transcriptional or posttranscriptional modulation of CeTwist function. Certainly a simple activation by physiological CeTwist levels would result in activation throughout the $\mathrm{M}$ lineage and loss of numerous nonuterine cell fates.

Once the segregation of uterine and vulval muscle precursors has occurred, a second level of regulation of $m l s-1$ activity is evident. Expression of $m l s-1$ reporters at this stage is observed in one of two vulval muscle types (the vm2 cells). MLS-1 appears not to be essential for development or activity of these cells, because mls-1 mutant animals differentiate both types of vulval muscles and show egg-laying function. Instead, it seems reasonable to propose that $\mathrm{mls}-1$ expression in this set of cells is tolerated as a consequence of previous commitment processes that may block the uterine-musclespecifying effect of MLS-1, presumably at a posttranslational level (e.g., through lack of essential cofactors).

\section{T-Box factors and mesodermal cell-type specification}

The function of mls-1 in mesodermal specification resembles that of a diverse class of Drosophila genes termed muscle founder identity genes. Choices between muscle fates in Drosophila are the result of an asymmetric cell division characterized by the combination of loss or maintenance of expression of these identity genes (for review, see Baylies et al. 1998). Identity gene mutations often transform cells to adopt sister cell fates, whereas ectopic expression can produce the reverse transformation. We found a similar pattern of transformations between sister muscle precursors in mls-1 mutant animals. None of the four characterized Drosophila T-box genes are known to be muscle founder identity genes; none has been shown to produce a simple late-lineage cell fate transformation in the mesoderm, although mutations in Drosophila brachyenteron (byn) show loss of longitudinal muscle fibers surrounding the midgut (Kusch and Reuter 1999). It is not clear if byn defects are the result of direct involvement in longitudinal muscle fibers or indirect effects on other tissue.

Given that T-box factors are conserved throughout animal phylogeny and have been implicated in a wide variety of developmental control processes, it seems a reasonable goal to draw both structural and functional parallels between specific family members within or between species. There are two rather substantial challenges in providing such a global understanding of the protein family, however. First, there is a need to draw a tree depicting evolutionary and structural relatedness within the family. The challenges in drawing such a tree are both theoretical and statistical. The interpretation of historical (and often stochastic) amino acid substitution events on a probabilistic and functional level from the sequence alone leads to inevitable uncertainty. In the case of the T-box family and MLS-1, there is very strong statistical support for a defined subfamily (with mouse TBX1 as a canonical member) that also contains representatives from humans and Drosophila and at least three additional C. elegans members. Degrees of relatedness within this subfamily become more problematic: a published tree using a distance matrix based on the neighbor-joining method suggests that the mls-1 product is the closest C. elegans homolog of TBX1 (Ruvkun and Hobert 1998). Using a variety of algorithms and parameters for cladistic analysis, we found that this assignment is highly dependent on the process used to construct the tree (S. Kostas, unpubl.). Importantly, all of the algorithms and parameters that we tested place MLS-1, MAB-9 (Woollard and Hodgkin 2000), TBX1 (Chieffo et al. 1997), and a small set of additional T-box domains in a clear subfamily.

Several genetic observations on mouse and human TBX1 evidence critical roles for this factor in vertebrate development. Mouse TBX1 is expressed in a number of tissues, including tongue muscle and cardiovascular mesenchymal tissues (Chapman et al. 1996; Jerome and Papaioannou 2001; Lindsay et al. 2001; Merscher et al. 2001). Human TBX1 has been shown to localize to a region of Chromosome $22(22 \mathrm{q} 11)$ that is deleted in velocardiofacial/DiGeorge syndrome (DGS; Chieffo et al. 1997). DGS is characterized by cardiac anomalies, hypoplasia or aplasia of the thymus and parathyroid glands, and mild craniofacial dysmorphia (DiGeorge 1965; Conley et al. 1979). Tbx1 knockout mice show striking phenocopy of velocardiofacial/DiGeorge syndrome, suggesting that TBX1 may account for many of the DGS-associated defects. The role of TBX1 in heart development is not understood.

The second challenge in constructing a orthologous structure/function compilation is how to compare spatial patterns (sites of action and mutant phenotypes) between different phyla. An understanding of correspondences between cell types and morphological structures over evolutionary time can be a difficult prospect. Some tissues (such as striated muscle) appear structurally and functionally conserved throughout evolution and are easily compared across phyla; other tissues such as uterine muscle have a less clearly defined phylogenetic homology. Comparisons of data from invertebrate and vertebrate genetics also involve an operational challenge. In C. elegans and Drosophila, developmental phenotypes can be characterized at the level of single cell fate alterations. In vertebrate systems, the characterization has more frequently focused on overall tissue morphologies; given the regulative nature of vertebrate development, such alterations are frequently several steps removed from the initial (cell-intrinsic) mutational effect. As parallel analysis of TBX1 family function progresses, hypotheses for phylogenetic correspondence at the cell and tissue level will certainly emerge.

Our work on mls-1 (that it is responsible for a transformation of fates between sister cells) and a recent study by Woollard and Hodgkin (2000) of mutations in mab-9 (which produce fate transformations between ad- 
jacent cells| provide a hypothesis for vertebrate TBX1 function that should be testable in the long term: the complex phenotype seen in the DiGeorge syndrome (and potentially in other TBX1-affecting mutations) could be due to a simple set of direct cell fate transformations. Given the plethora of cell and tissue interactions in vertebrates, it could reasonably be expected that such transformations would lead to other derangement and produce the complex set of phenotypes observed in the final outcome. The validity of this hypothesis should become clear as the cellular focus of action and earliest defects in the vertebrate $t b x$ mutations are elucidated.

\section{Materials and methods}

\section{C. elegans strains}

Analyses were performed at $22^{\circ} \mathrm{C}-23^{\circ} \mathrm{C}$, unless otherwise noted. The alleles used in this work are: unc-32(e189), dpy18(e499), dpy-17(e164), unc-36(e251), sma-4(e729), sma3(e491) (Brenner 1974), mec-14(u296) (gift of G. Caldwell and M. Chalfie, Columbia University, New York, NY), hlh8(nr2061) (Corsi et al. 2000), lin-39 (n1760) (Clark et al. 1993).

Strains carrying integrated cell-type specific reporter transgenes were used to facilitate identification of specific cell fates within the M lineage:

myo-3::gfp: PD4251(ccIs4251)I, active in all body-wall and vulval muscles (Fire et al. 1998).

hlh-8::gfp: PD4666(ayIs6)X and PD4667|ayIs7|IV, active in all undifferentiated cells in the M lineage (Harfe et al. 1998b).

eg1-15::gfp: NH2447(ayIs2)IV, active in adult vm1 muscles (Harfe et al. 1998a). Nde-box::gfp, PD4655(ccIs4655)II and PD4297(ccIs4297), active in eight vulval and eight uterine muscles, and the enteric muscles (Harfe et al. 1998b; S. Kostas and A. Fire, unpubl.).

myo-3::secreted gfp: (secreted gfp), GS1919(arIs37)I and GS2077(arIs39|X, used to visualize coelomocytes (gift of J. Fares and I. Greenwald, Columbia University, New York, NY).

arg-1::gfp: PD4443(ccIs4443)IV, active in adult vulval muscles, enteric muscles, and the head mesodermal cell (Mello et al. 1994; A. Melendez, I. Greenwald, K. Liu, S. Kostas, and A. Fire, unpubl.).

ceh-24::gfp: PD4595(ccIs4595)V, active in adult vulval muscles, pharyngeal muscle $\mathrm{m} 8$, and in a set of neurons in the head (Harfe and Fire 1998).

intrinsic CC::gfp: PD4439(ccIs4439)III, active in embryonic and postembryonic coelomocytes (Harfe et al. 1998b; J. Fares, S. Kostas, and A. Fire, unpubl.).

rgs-2::gfp: LX354 IV, strongly active in uterine muscles, less active in some neurons, vulval muscles, and enteric muscles (Dong et al. 2000).

\section{Screen for altered pattern of myo-3::gfp expression}

PD4251 animals carrying an integrated myo-3::gfp transgene were mutagenized with EMS, and nonclonal $\mathrm{F}_{2}$ and $\mathrm{F}_{3}$ populations were observed by direct fluorescent illumination on a grid slide (KR-834 Reticle Slide, Klarmann Rulings) in $330 \mu \mathrm{M}$ levamisole. Vulval muscles and one body-wall muscle quadrant were counted in each adult and L4, and animals with altered patterns were recovered for further analysis. From $~ 7000$ haploid genomes, we identified 8 independent mutations with altered muscle lineages. Five mutations had low penetrance defects earlier in the M lineage and were not further characterized; three mutations (cc569, cc570, and cc571) were highly penetrant, recessive, and failed to complement, defining the mls-1 locus.

An important element of this screen (in comparison with earlier screens for mutants affecting sex muscles) was the lack of any specific selection for defects in function of the egg-laying muscles (Egl phenotypes, e.g., Trent et al. 1983). Indeed, the mls-1 mutations were each egg-laying competent. Analysis of mls-1(cc569) indicated that both the stage at which the first eggs are laid and the accumulation of eggs in the uterus after 24 $\mathrm{h}$ were comparable to wild type (data not shown).

\section{Genetic and molecular analysis of mls-1}

Mapping and complementation analysis of mls-1 was carried out with allele cc569 using standard methods with the appearance of ectopic vulval muscles in an egl-15::gfp (ayIs2) background as an assay. No evidence of maternal effect was observed. Three-factor mapping was carried out using mec14(u296) unc-32(e189)/mls-1(cc569); ayIs2 and sma-3(e491) lin39(n1490)/mls-1(cc569); ayIs2. 2/11 of the Mec-non-Dpy and $16 / 19$ of the Sma-non-Lin acquired $\mathrm{mls}-1$, indicating a locus between mec-14 and lin-39. cc570 and cc571 were also mapped and found to reside in the central portion of Chromosome III (three-factor mapping using sma-3 and unc-32).

Rescue of mls-1 mutants was assayed in transgenic lines derived from mls-1(cc570); egl-15::gfp (ayIs2). For each DNA mixture, several independent transgenic lines were recovered and scored for restoration of the wild-type egl-15::gfp pattern. Whole-genome DNA from YAC-bearing yeast strains $(50 \mu \mathrm{g} /$ $\mathrm{mL})$ was injected with plasmid pRF4 $(100 \mu \mathrm{g} / \mathrm{mL})$ to identify transformed lines (Mello et al. 1991). After identification of a single rescuing YAC (Y69F12), fosmids and cosmids in this region were injected with pRF4 $(10 \mu \mathrm{g} / \mathrm{mL})$. Fosmid H14A12 rescued all alleles of $\mathrm{mls}-1$.

\section{Derivatives of the mls-1 gene used in microinjection/rescue} assays

To delineate the mls-1 gene, plasmids with the following inserts were assayed for rescue of $\mathrm{mls}-1$ (cc570).

pSAK84: An 8.4-kb HindIII-NcoI fragment from H14A12 contained at least two predicted coding sequences (H14A12.4 and H14A12.3).

pSAK89: AgeI digestion of pSAK84 followed by T4 DNA polymerase treatment and religation produced a four-base insertion. The modified gene would be predicted to produce a protein with the first 104 amino acids of H14A12.4 followed by five missense amino acids and a stop codon.

pSAK107: A 4.4-kb BgIII-PmeI fragment contained the predicted coding region of gene H14A12.4 with 1340 bases of upstream sequence and 1668 bases of downstream sequence. No other predicted gene overlaps with this fragment.

pSAK244: A 4.3-kb mls-1 fragment with 1320 bases of upstream sequence and 1491 bases of downstream sequence was PCR-amplified from wild-type DNA.

pSAK246: An equivalent fragment to pSAK244 was amplified from mls-1(cc570).

pSAK534: A mls-1::1acZ reporter was generated by inserting lac $Z$ sequences (from pPD95.57) into the mls-1 coding region (from pSAK107). lacZ was fused 6 amino acids from the $\mathrm{C}$ terminus of $m l s-1$; the last 6 amino acids (out of frame) and $m l s-1$ 3 ' sequence are downstream of lacZ.

pSAK598, pSAK601, pSAK604: pSAK534 was modified to change the -179 E-box to CAGCTG, CAGCTG, and ATCGAT, respectively. 
pSAK633: mls-1 coding sequence and $1.6 \mathrm{~kb}$ downstream were ligated downstream of the hlh-8/CeTwist promoter in pJKL502 (gift of J. Liu, Cornell University, Ithaca, NY).

\section{Induction of expression by heat shock}

The heat shock (hs)-inducible constructs used were hs::hlh-8 (pBH48.8) and hs::hlh-2 (pKM1035; Harfe et al. 1998b). A mixed stage population of animals was heat shocked at $37^{\circ} \mathrm{C}$ for $1 \mathrm{~h}$ and allowed to recover for $6 \mathrm{~h}$ at $25^{\circ} \mathrm{C}$ prior to staining for $\beta$-galactosidase activity.

\section{RNAi}

dsRNA (produced from a 549-bp genomic fragment spanning mls-1 exons 3-5) was injected into animals with cell-type-specific integrated $g f p$ reporters, and progeny were scored for muscle defects (Fire et al. 1998). In addition, L1 larvae were soaked overnight in the dsRNA, with a small aliquot of OP50 bacteria added (Tabara et al. 1998). Animals were removed from dsRNA, cultured for $2 \mathrm{~d}$, and examined for viability and muscle specification defects.

\section{Acknowledgments}

We thank K. Liu, A. Corsi, M. Krause, A. Melendez, I. Greenwald, C. Mello, M. Dong, M. Koelle, C. Fan, A. Coulson, D. Fitch, E. Hedgecock, M. Halpern, B. Harfe, J. Yanowitz, L. Timmons, J. Hsieh, B. Kelly, S. Xu, J. Fleenor, S. Parrish, R. Alcazar, M. Etcheverry, J. Moreno, and our colleagues at Carnegie Institution for help and suggestions. Some nematode strains used in this work were provided by the Caenorhabditis Genetics Center, which is supported by NIH's National Center for Research Resources. This work was supported by the NIH (grants R01GM37706 to A.F. and T32GM07231 to S.K.).

The publication costs of this article were defrayed in part by payment of page charges. This article must therefore be hereby marked "advertisement" in accordance with 18 USC section 1734 solely to indicate this fact.

\section{References}

Ardizzi, J. and Epstein, H. 1987. Immunochemical localization of myosin heavy chain isoforms and paramyosin in developmentally and structurally diverse muscle cell types of the nematode Caenorhabditis elegans. J. Cell Biol. 105: 2763 2770.

Arnold, H. and Braun, T. 2000. Genetics of muscle determination and development. Curr. Top. Dev. Biol. 48: 129-164.

Bate, M., Rushton, E., and Currie, D. 1991. Cells with persistent twist expression are the embryonic precursors of adult muscles in Drosophila. Development 113: 79-89.

Baylies, M., Bate, M., and Ruiz Gomez, M. 1998. Myogenesis: A view from Drosophila. Cell 93: 921-927.

Beddington, R., Rashbass, P., and Wilson, V. 1992. Brachyury-A gene affecting mouse gastrulation and early organogenesis. Dev. Suppl. 117: 157-165.

Brenner, S. 1974. The genetics of Caenorhabditis elegans. Genetics 77: 71-94.

Chapman, D., Garvey, N., Hancock, S., Alexiou, M., Agulnik, S., Gibson-Brown, J., Cebra-Thomas, J., Bollag, R., Silver, L., and Papaioannou, V. 1996. Expression of the T-box family genes, Tbx1-Tbx5, during early mouse development. Dev. Dyn. 206: 379-390.
Chieffo, C., Garvey, N., Gong, W., Roe, B., Zhang, G., Silver, L., Emanuel, B., and Budarf, M. 1997. Isolation and characterization of a gene from the DiGeorge chromosomal region homologous to the mouse Tbx1 gene. Genomics 43: 267277.

Clark, S., Chisholm, A., and Horvitz, H. 1993. Control of cell fates in the central body region of $C$. elegans by the homeobox gene lin-39. Cell 74: 43-55.

Conley, M., Beckwith, J., Mancer, J., and Tenckhoff, L. 1979. The spectrum of the DiGeorge syndrome. J. Pediatr. 94: 883 890.

Consortium. 1998. Genome sequence of the nematode C. elegans: A platform for investigating biology. Science 282: 2012-2018.

Corsi, A., Kostas, S., Fire, A., and Krause, M. 2000. Caenorhabditis elegans twist plays an essential role in non-striated muscle development. Development 127: 2041-2051.

DeVore, D., Horvitz, H., and Stern, M. 1995. An FGF receptor signaling pathway is required for the normal cell migrations of the sex myoblasts in C. elegans hermaphrodites. Cell 83: 611-620.

DiGeorge, A. 1965. Discussion on a new concept of the cellular basis of immunology. J. Pediatr. 67: 907.

Dong, M., Chase, D., Patikoglou, G., and Koelle, M. 2000. Multiple RGS proteins alter neural $\mathrm{G}$ protein signaling to allow C. elegans to rapidly change behavior when fed. Genes \& Dev. 14: 2003-2014.

Fire, A., Xu, S., Montgomery, M., Kostas, S., Driver, S., and Mello, C. 1998. Potent and specific genetic interference by double-stranded RNA in Caenorhabditis elegans. Nature 391: 806-811.

Fitzgerald, K. and Greenwald, I. 1995. Interchangeability of Caenorhabditis elegans DSL proteins and intrinsic signalling activity of their extracellular domains in vivo. Development 121: $4275-4282$.

Greenwald, I., Sternberg, P., and Horvitz, H. 1983. The lin-12 locus specifies cells fates in Caenorhabditis elegans. Cell 34: 435-444.

Harfe, B. and Fire, A. 1998. Muscle and nerve-specific regulation of a novel NK-2 class homeodomain factor in Caenorhabditis elegans. Development 125: 421-429.

Harfe, B., Branda, C., Krause, M., Stern, M., and Fire, A. 1998a. MyoD and the specification of muscle and non-muscle fates during postembryonic development of the C. elegans mesoderm. Development 125: 2479-2488.

Harfe, B., Vaz Gomes, A., Kenyon, C., Liu, J., Krause, M., and Fire, A. 1998b. Analysis of a Caenorhabditis elegans Twist homolog identifies conserved and divergent aspects of mesodermal patterning. Genes \& Dev. 12: 2623-2635.

Henikoff, S. and Henikoff, J. 1992. Amino acid substitution matrices from protein blocks. Proc. Natl. Acad. Sci. 89: 1091510919.

Herrmann, B., Labeit, S., Poustka, A., King, T., and Lehrach, H. 1990. Cloning of the $\mathrm{T}$ gene required in mesoderm formation in the mouse. Nature 343: 617-622.

Hsieh, J., Liu, J., Kostas, S., Chang, C., Sternberg, P., and Fire, A. 1999. The RING finger/B-Box factor TAM-1 and a retinoblastoma-like protein LIN-35 modulate context-dependent gene silencing in Caenorhabditis elegans. Genes \& Dev. 13: $2958-2970$.

Jerome, L. and Papaioannou, V. 2001. DiGeorge syndrome phenotype in mice mutant for the T-box gene, Tbx1. Nat. Genet. 27: 286-291

Kelly, W., Xu, S., Montgomery, M., and Fire, A. 1997. Distinct requirements for somatic and germline expression of a generally expressed Caenorhabditis elegans gene. Genetics 
146: $227-238$.

Kenyon, C. 1986. A gene involved in the development of the posterior body region of C. elegans. Cell 46: 477-487.

Krause, M., Fire, A., Harrison, S., Priess, J., and Weintraub, H. 1990. CeMyoD accumulation defines the body wall muscle cell fate during C. elegans embryogenesis. Cell 63: 907-919.

Krause, M., Park, M., Zhang, J., Yuan, J., Harfe, B., Xu, S., Greenwald, I., Cole, M., Paterson, B., and Fire, A. 1997. A C. elegans E/Daughterless bHLH protein marks neuronal but not striated muscle development. Development 124: 2179-2189.

Kusch, T. and Reuter, R. 1999. Functions for Drosophila brachyenteron and forkhead in mesoderm specification and cell signalling. Development 126: 3991-4003.

Lindsay, E., Vitelli, F., Su, H., Morishima, M., Huynh, T., Pramparo, T., Jurecic, V., Ogunrinu, G., Sutherland, H., Scambler, P., et al. 2001. Tbx1 haploinsufficiency in the DiGeorge syndrome region causes aortic arch defects in mice. Nature 410: $97-101$.

Liu, J. and Fire, A. 2000. Overlapping roles of two Hox genes and the exd ortholog ceh-20 in diversification of the C. elegans postembryonic mesoderm. Development 127: 5179-5190.

Mello, C., Kramer, J., Stinchcomb, D., and Ambros, V. 1991. Efficient gene transfer in C.elegans: Extrachromosomal maintenance and integration of transforming sequences. EMBO J. 10: 3959-3970.

Mello, C., Draper, B., and Priess, J. 1994. The maternal genes apx-1 and $g l p-1$ and establishment of dorsal-ventral polarity in the early C. elegans embryo. Cell 77: 95-106.

Merscher, S., Funke, B., Epstein, J., Heyer, J., Puech, A., Lu, M., Xavier, R., Demay, M., Russell, R., Factor, S., et al. 2001. TBX1 is responsible for cardiovascular defects in velo-cardiofacial/DiGeorge syndrome. Cell 104: 619-629.

Muller, C. and Herrmann, B. 1997. Crystallographic structure of the $\mathrm{T}$ domain-DNA complex of the Brachyury transcription factor. Nature 389: 884-888.

Nishida, H. and Satoh, N. 1983. Cell lineage analysis in ascidian embryos by intracellular injection of a tracer enzyme. I. Up to the eight-cell stage. Dev. Biol. 99: 382-394.

Okkema, P., White-Harrison, S., Plunger, V., Aryana, A., and Fire, A. 1993. Sequence requirements for myosin gene expression and regulation in C. elegans. Genetics 135: $385-$ 404.

Ordahl, C. and Le Douarin, N. 1992. Two myogenic lineages within the developing somite. Development 114: 339-353.

Perry, R. and Rudnick, M. 2000. Molecular mechanisms regulating myogenic determination and differentiation. Front. Biosci. 5: D750-D767.

Pflugfelder, G., Roth, H., and Poeck, B. 1992. A homology domain shared between Drosophila optomotor-blind and mouse Brachyury is involved in DNA binding. Biochem. Biophys. Res. Commun. 186: 918-925.

Ruvkun, G. and Hobert, O. 1998. The taxonomy of developmental control in Caenorhabditis elegans. Science 282: 2033 2041.

Schnabel, R. 1994. Autonomy and nonautonomy in cell fate specification of muscle in the Caenorhabditis elegans embryo: A reciprocal induction. Science 263: 1449-1452.

Smith, J. 1999. T-box genes: What they do and how they do it. Trends Genet. 15: 154-158.

Sulston J. and Horvitz H. 1977. Post-embryonic cell lineages of the nematode, Caenorhabditis elegans. Dev. Biol. 56: 110 156.

Sulston, J. and White, J. 1980. Regulation and cell autonomy during postembryonic development of Caenorhabditis elegans. Dev. Biol. 78: 577-597.
Sulston, J., Schierenberg, E., White, J., and Thomson, J. 1983. The embryonic cell lineage of the nematode Caenorhabditis elegans. Dev. Biol. 100: 64-119.

Tabara, H., Grishok, A., and Mello, C. 1998. RNAi in C. elegans: Soaking in the genome sequence. Science 282: 430431.

Thomas, J. 1990. Genetic analysis of defecation in Caenorhabditis elegans. Genetics 124: 855-872.

Trent, C., Tsung, N., and Horvitz, H. 1983. Egg-laying defective mutants of the nematode C. elegans. Genetics 104: 619-647.

Woollard, A. and Hodgkin, J. 2000. The Caenorhabditis elegans fate-determining gene mab-9 encodes a T-box protein required to pattern the posterior hindgut. Genes \& Dev. 14: 596-603. 


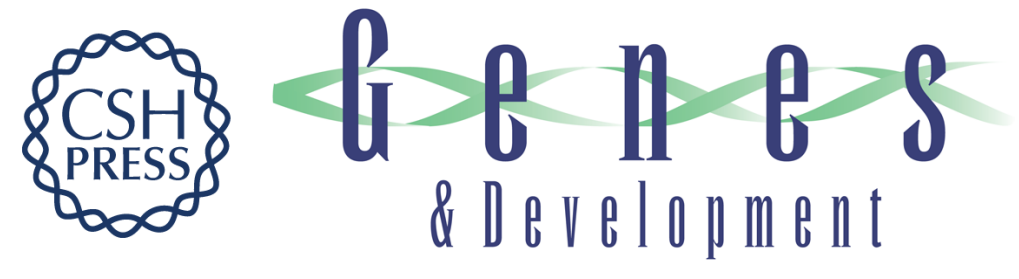

\section{The T-box factor MLS-1 acts as a molecular switch during specification of nonstriated muscle in C. elegans}

Stephen A. Kostas and Andrew Fire

Genes Dev. 2002, 16:

Access the most recent version at doi:10.1101/gad.923102

References This article cites 50 articles, 24 of which can be accessed free at: http://genesdev.cshlp.org/content/16/2/257.full.html\#ref-list-1

License

Email Alerting

Receive free email alerts when new articles cite this article - sign up in the box at the top Service right corner of the article or click here.

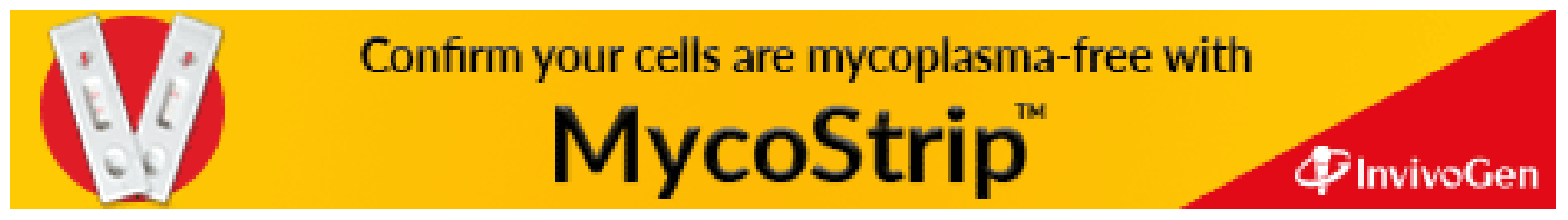

